\title{
Group Intelligence: a distributed cognition perspective
}

\author{
Osama Mansour \\ School of Mathematics and Systems \\ Engineering, Växjö University \\ Växjö, Sweden. \\ osama.mansour@vxu.se
}

\begin{abstract}
The question of whether intelligence can be attributed to groups or not has been raised in many scientific disciplines. In the field of computer-supported collaborative learning, this question has been examined to understand how computer-mediated environments can augment human cognition and learning on a group level. The era of social computing which represents the emergence of Web 2.0 collaborative technologies and social media has stimulated a wide discussion about collective intelligence and the global brain. This paper reviews the theory of distributed cognition in the light of these concepts in an attempt to analyze and understand the emergence process of intelligence that takes place in the context of computer-mediated collaborative and social media environments. It concludes by showing that the cognitive organization, which occurs within social interactions serves as a catalyst for intelligence to emerge on a group level. Also a process model has been developed to show the process of collaborative knowledge construction in Wikipedia that characterizes such cognitive organization.
\end{abstract}

Keywords: Group Intelligence, Theory of Distributed Cognition, Social Media, Web 2.0, Collaborative.

\section{INTRODUCTION}

Intelligence has always been a controversial term when discussed in the context of collaborative environments in an attempt to attribute it to groups. This is because it is habitual to attribute thoughts and intentions to individuals and to reduce group phenomenon into actions by the individual members of the group ([7], [9], [23]). In this paper, group intelligence refers to the aggregation of individuals' distributed cognitive processes over a common platform in the form of socially shared knowledge and meaning. It is opted that intelligence is a property that emerges during a group activity and thus it is an attribute on a group level. The paper uses the term group intelligence rather than collective, collected intelligence or any other terms based on the idea that all these terminologies refer to intelligence that takes place during a social group activity.

The current era of social computing which represents the emergence of an assortment of social media applications that support collaboration, knowledge sharing, social networking, and self-organization ([5], [17], [20], [25], [30]) has stimulated a wide discussion about concepts like collective intelligence, crowdsourcing, global brain, etc ([15], [24], [25]). Consequently, there is a need for a profound understanding and examination of how intelligence can be achieved by groups supported by social media technologies. At this respect, distributed cognition (c.f. section II) has been a focus by researchers where they discussed different aspects of intelligence that emerge within the social interactions of group members (e.g. [9], [23], [26]). In this sense, the use of the distributed cognition approach can be found in the fields of semantic web, distributed artificial intelligence, and ambient intelligence [7]. Distributed cognition means cognitive processes that are distributed across the members of a social group [9]. These cognitive processes are involved in memory, decisionmaking, inference, reasoning, learning, etc which sum up human intellectuality [10]. A collaborative system can be viewed as - a large cognitive system involving multiple people interacting with each other and a wide range of artifacts to perform an activity [21]. Hence with the increasing developments in collaborative and knowledge sharing technologies [25], the focus on group intelligence is a compelling issue to explore how state-of-the-art technologies may influence human cognition. Therefore the paper tries to examine how social media and collaborative technologies mediate cognitive processes of a group of people and the influence on the emergent intelligence. A thorough review of the theory of distributed cognition has been conducted and different aspects of the theory have been examined to show how cognitive processes take place within a group activity supported by social media technologies. This will contribute to understanding how cognition is distributed and shared among groups and the influence of the flow of cognitive processes on intelligence.

\section{THEORY OF DISTRIBUTED COGNITION}

Edwin Hutchins has developed the theory of distributed cognition in a simulation experiment of an airline cockpit (c.f. [10]). In this experiment, Hutchins views the cockpit as a large cognitive system with emergent cognitive properties. These properties emerge as a result of interactions of the crewmembers, interactions between the crew and the artifacts, coordination mechanisms, and the propagation of information in the form of representational states (i.e. mental state, state of knowledge) across representational media that is the main concern of the theory ([8], [10], [21]). The propagation of information in the form of representational states refers to the transformation of information during the conduct of a group activity [21]. Representational media is located within individuals (e.g. memory, knowledge, skills,), within group members (e.g. shared meanings), and in the physical structure (e.g. tools such as social media) ([8], [9]). These representational media have different properties that constraint the required cognitive processes to communicate representational states [10]. Therefore, the emphasis is on the propagation of information in the distributed cognition theory as this process entails group meanings, which form the emergent properties of the system ([8], [10], [23]). In this sense, Rogers [21] explained that the distributed cognition approach seeks to explicate the complex interdependencies between people and artifacts in their work activities, of which an important part is identifying the problems, breakdowns and the distributed problem solving processes that emerge to deal with them. 
Hutchins and Klausen [10] emphasized the importance of the distributional characteristics of information, which are essential to maintain intersubjective understandings and shared meanings. Intersubjective understanding emerges when individuals' knowledge gets shared during a group activity to form a shared resource for their distributed negotiations, interactions, and the development of shared meanings ([9], [23]). At this respect, a major aspect of this theory is that it moves beyond understanding individual cognitive processes to reach an understanding of cognition that occurs on a system or a group level ([8], [9], [19], [21], [22]). Therefore, the distributed cognition approach takes the whole system as a unit of analysis rather than studying the components of the system ([9], [19], [21]). Stahl [23] reflected on the issue of the unit of analysis as methodological rather than ontological where the focus should be the group rather than the individual member of the group. Three kinds of distribution of cognitive processes are involved with the theory of distributed cognition: cognitive processes distributed across the members of a social group, cognitive processes may involve coordination between external and internal (material and environmental) structure, and processes maybe distributed through time ([8], [9]). To sum up, distributed cognition approach seeks to understand the organization of large cognitive systems, which could be seen as sort of cognitive architecture on a group level. To understand intelligence, we need to consider such larger systems where the parts get assembled together to achieve tasks and develop shared meanings [9]. These tasks and shared meanings could not be achieved by a sole individual and may influence human cognition on a group or community level ([9], [26]), which therefore impact their ability to collectively solve problems and make decisions.

\section{GROUP INTELLIGENCE}

The question of how cognition could be examined on a group level has been raised in many scientific disciplines such as social psychology and cognitive science ([7], [10], [23]). The traditional conceptions that view cognition as limited to the individual mind and that learning and thinking only occurs on an individual level, have led to difficulties in understanding intelligence on a group level ([1], [7], [9], [23]). However, there is an increasing number of approaches proposing that human cognition is not solely found inside an individual brain but also involves interactions with other minds ([7], [8], [21], [22]). These approaches are focused on the cognition of larger systems that emerge on a group level. Theories of distributed cognition, extended mind, and situatedness are focusing on the extension of individual cognition to involve interconnections with other minds and the ability to form patterncompleting and pattern-creating actions [6]. In this sense, distributed cognition is a confluence with collective or group intelligence and cognition could be understood as collective intelligence that is cognitive processes and structures that emerge at the social level [7].

In contrast to traditional conceptions of learning and thinking, many researchers viewed knowledge as a result from social construction that is achieved by individuals rather merely individual observation ([1], [7], [22], [23]). This has led to the idea that individuals learn due to social interactions with other individuals in the course of a group activity [23]. A simple example to clarify this is about a newborn learning child. In the early childhood, a child starts interacting with adults and artifacts around him. Over time, the child starts to learn and develop new functional skills that would allow him to function in the absence of adults. Therefore, learning could be seen as interactional. At this respect, Argyris and Schön [1] mentioned "Learning is a term applicable to individuals within the context of a group, but when individuals learn to interact with one another so as to carry out shared tasks, one can speak of the group itself as learning" (p. 322). In this view of interactional learning, the source of individual knowledge is the group. Stahl [23] provided a number of views on learning from the field of computer-supported collaborative learning (CSCL) that represent diverse sources of knowledge and meanings both from individuals and groups. These different views of learning may provide some perspectives on the propagation of information both internally inside an individual mind or externally through the interaction among individuals and with physical artifacts, which is central to the theory of distributed cognition. In this sense, Smith [22] argued that groups have more potential to remember information rather than individuals due to transactive memory creating a larger distributed memory capacity than an individual can possess. Also, he reflected that the communication and distribution of information among group members helps to effectively search for diverse information which can potentially improve the overall group performance. As a result, cognition emerges on a group level due to the distribution and communication of knowledge between group members, which may result an amplified cognition that is not reducible to the individual mind. Further, the emergent group cognition consists of group memory, group problem solving, and group decisionmaking abilities, which form the basis for non-reducible group intelligence to individuals. However, in order to achieve and maintain group intelligence, there is a need for an effective communication and collaboration media so that cognition is distributed and shared by groups. The next section discusses different aspects of Web 2.0 and social media technologies, which may provide platforms for the propagation and communication of information.

\section{WEB 2.0 AND SOCIAL MEDIA}

It might be said that the vision of the global brain has been reached in this current era of social computing. The global brain is an intelligent network formed by the people of this planet together with the knowledge and communication technologies that connect them together [7]. This vision is manifested in the application of a variety of Web 2.0 and social media technologies. Web 2.0 is a generic term that refers to a variety of social computing applications such as Wikis, blogs, peer-to-peer downloading, social networking, etc ([17], [25]). The underlying concepts of Web 2.0 technologies involve the architecture of participation, collaborative knowledge construction, network as a platform, consuming and remixing data from multiple sources, etc ([2], [6], [11], [14], [17], [20], [25]). The significant impact of Web 2.0 technologies lies in connecting large numbers of people and in their flexibility that allows people to appropriate their features in a way that enable them to link and share their individual tacit knowledge, which represent individual cognitive processes, and align this knowledge with the social context of producing group knowledge [13]. This knowledge is not reducible to individuals as it contains multiple ideas and interpretations that have been processed and manipulated by other people. Many researchers have reflected on the capabilities of Web 2.0 or social web and the potential for augmented social cognition, collective intelligence, and wisdom of crowds ([2], [6], [20], [24], [25], [27]). Web 2.0 environments reflect the concept of networks of practice. Electronic and networked communities of practice represent large applications of Web 2.0 and social media technologies with their rich knowledge exchange and social interactions. Wasko and 
Faraj [28] defined electronic networks of practice as "a selforganizing, open activity system focused on a shared practice that exists primarily through computer-mediated communication." (p. 3 ). In this sense, Web 2.0 environments support large number of individuals who voluntarily choose to be part of online social communities and engage in open activities where they share their knowledge to help solve problems of common interests. The properties of these technologies reflect a major notion, which is the propagation and distribution of shared knowledge within social interactions. This notion is implied in the theory of distributed cognition where group cognition takes the form of shared knowledge. In this sense, Touzet [26] explained, “...we can just about to conceive distributed cognition as sum of contextual intelligences operating as a whole structure." Based on the conceptions of Web 2.0, a Web 2.0 collaborative system could be seen as a single and large structure integrating multiple groups and communities with common interests where they communicate and share their knowledge. This results cognitive processes of these groups to be distributed and are manifested in collaborative knowledge. Collaborative knowledge is rich of multiple ideas, meanings, and interpretations, which externalize the contents of thought, and is embodied in the coordination of our identities in a social and collaborative context [22]. Consequently, Web 2.0 environments mediate the distribution of our cognitive processes and support our social interactions in a sense that the knowledge transformed across members of the groups, from a distributed cognition perspective, can be viewed as a form of group intelligence. Such group or social Intelligence can result from social learning that is interactional, which in turn illuminate augmented social cognitive processes.

The next section discusses Wikipedia, which is based on the Wiki technology and has been rising as the largest online encyclopedia ([4], [12], [15]) to explain the processes of largescale collaborative knowledge construction and decision-making.

\section{A. Wikipedia: A Process Model}

The Wiki technology refers to a simple website that enables everyone to collaboratively create, edit, tag, and link content ([11], [12], [16], [25]).

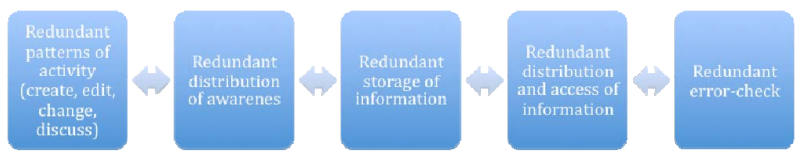

Figure 1. Process model describing the processes of collaborative knowledge construction in Wikipedia.

In this sense, articles in Wikipedia are collaboratively created and content is refined and filtered in an iterative manner. Everyone can contribute knowledge to a particular subject ([12], [16]). Other people may change or edit the content according to their experiences in each subject. Continuously, this iterative process of refinement embodies social knowledge that is communicated and shared with self-organized communities of Wikipedians involving decision-making processes especially when topics are controversial. Topics that are controversial require collaborative assessment and consensus, which may involve argumentations and discussions over the Wiki until a peer-reviewed article is reached. Any changes to the content can be seen by group members and are aware of them. Such collaborative process of creating and communicating content in Wikipedia embodies different aspects of the theory of distributed cognition (see Figure
1) and every aspect is cognitive-ingrained. Figure 1 above visualizes theoretical aspects of the theory of distributed cognition that represent sequential and iterative processes of collaborative knowledge construction in Wikipedia. The process model describes processes that take place during a cognitive activity, which is continuously executed by a group of people for collaborative knowledge construction. These processes entail propagation of multiple states of people. People are performing these processes iteratively. Each process is associated and altered by cognitive abilities of everyone resulting cognitive processes to be distributed across the group. Such distribution characterizes a kind of cognitive organization, which is created and maintained through social interactions and connectivity that are represented by arrows in the process model. The iteration and recurring efforts in the process of collaborative knowledge construction in Wikipedia create a cognitive feedback loop leading to some sort of organization. According to Robert (1964 cited in [9], p. 2) that "such social organization could be seen as a sort of architecture of cognition at the community level". This iterative process and cognitive organization in Wikipedia implies a kind of - social capital, which is embedded within networks of social structure ([18], [28]). Social capital is a term used in community studies, which highlights the central importance of networks of relationships that provide a basis for trust, cooperation, and collective action (ibid). Nahapiet and Ghoshal [18] argued that social capital facilitates the creation of intellectual capital based on the idea that networks of relationships constitute a resource for the social conduct and provide the members of the groups with collectivity-owned capital (Buordieu, 1986, p. 249 cited in [18]). In this sense, they referred to intellectual capital as "...the knowledge and knowing capability of a social collectivity, such as an organization, intellectual community, or professional practice." (ibid, p. 5). The community of Wikipedians is supported by the Wiki technology that constitutes the structure or the platform for their social interactions and collective actions. This helps to maintain the growth of social capital through enabling knowledge exchange and intellect leverage that is represented and combined in large numbers of articles. The cognitive characteristics of the community represent its memory capacity and the ability to manage and retrieve information, which can be determined by looking at what information is there, where it is located and how it moves in the community (ibid). In this sense, figure 1 represents a sequence of representational states leading to movement of information in Wikipedia. Hutchins [9] explained that such movement of information has an impact on the organizing behavior and coordination of the group and also depends on their assessment of own states of knowledge and others. Also, repeated patterns of activity may lead to the consolidation of functional assemblages and the result can be individual learning or organizational learning [9]. He further added that an important property of aggregate systems is that they may give rise to forms of organization that cannot develop in the component parts. Hence one may argue that the cognitive organization is by itself an intelligent effort enabled by Web 2.0 environments and also serves as a catalyst for intelligence to emerge on a group level.

\section{CONCLUSIONS}

In this paper, the theory of distributed cognition has been examined in the context of computer-mediated environments in an attempt to address the question of how intelligence can emerge on a group level. The theory provides some perspectives on the emergence process of intelligence through examining the 
distribution of cognitive processes across the members of social groups. Particularly, the theory has been examined in Web 2.0 environments, which entail rich social interactions and cognitiveingrained collaborative processes. The cognitive organization that is achieved through the propagation of knowledge, division of labor, and ongoing social interactions across the group results augmented social cognition and therefore serves as a catalyst for intelligence to emerge on a group level. This cognitive organization results due to the distribution of cognitive processes and is manifested in collaborative knowledge. The resulted collaborative knowledge is rich of multiple ideas, meanings, and interpretations of everyone within the group, which helps to enhance the ability of making decisions and solve problems and is not reducible to any of the members of the group. Furthermore, in order to provide an example of cognitive organization, a process model has been developed that describes the different processes involved in collaborative knowledge construction in Wikipedia. The model visualizes different theoretical aspects of the theory of distributed cognition. It also shows how different cognitiveingrained states propagate throughout the whole process creating a cognitive feedback loop leading to architecture of cognition at the community level.

\section{FURTHER RESEARCH}

Theories of social psychology and cognitive science are of great benefit and importance for the communities of CSCW and CSCL. Such theories provide diverse and enlightening perspectives of group dynamics, adaptive behavior, networks of social relationships, and studies of mind. These perspectives are essential to enhance our understanding of collaborative and group processes especially with increasing developments of computing technologies that facilitate social interactions and group work. Therefore, further research stemming from these theories is needed to further increase our understanding of group dynamics. In particular, these theories are useful to explore more about group and collective intelligence, which are prevailing concepts in the era of social computing.

\section{References}

[1] Argyris, C., \& Schön, D. (1978) Organizational learning: A theory of action perspective, Reading, Mass: Addison Wesley.

[2] Chi, E. (2008). The social web: research and opportunities. IEEE Computer. Vol. 41, pp. 88-91.

[3] Flor, N.V., Hutchins, E. (1992). Analyzing Distributed Cognition in Software Teams: a Case Study of Collaborative Programming During Adaptive Software Maintenance. In Empirical Studies of Programmers: Fourth Workshop, eds. J. Koenemann-Belliveau, T.

[4] Gloor, P., Copper, S. (2007). The new principles of swarm business. MITSloan Management Review. Vol. 48, pp. 81-84.

[5] Grossman, M., McCarthy, R. (2007). Web 2.0: is the Enterprise ready for the adventure? Issues in Information Systems. Vol. VIII. No. 2, pp. 180-185.

[6] Gruber, T. (2008). Collective knowledge systems: where the social web meets the semantic web. Web Semantics: Science, Services and Agents on the World Wide Web. Vol. 6, pp. 4-13.

[7] Heylighen, F., Heath, M., Van Overwalle, F. (2004) The emergence of distributed cognition: a conceptual framework. Proceeding of Collective Intentionality IV, Seina (Italy).

[8] Hollan, J., Hutchins, E., Kirsch, D. (2000). Distributed Cognition: Toward a New Foundation for Human-Computer Interaction Research ACM Transactions on HCI, Vol. 7, pp. 174196.
[9] Hutchins, E. (2000) Distributed Cognition. IESBS.

[10] Hutchins, E. \& Klausen, T. (1996). 'Distributed Cognition in an Airline Cockpit'. In Middleton, D, Engeström, Y. Communication and Cognition at Work. Cambridge: Cambridge University Press, 15-34.

[11] Kane, G., Fichman, R. (2009). The Shoemaker's Children: Using Wikis for Information Systems Teaching, Research, and Publication. MIS Quarterly, Vol. 33, pp. 1-22.

[12] Lee, M., Lan Y. (2007). From Web 2.0 to conversational Knowledge Management: Towards Collaborative Intelligence. Journal of Entrepreneurship Research. Vol. 2, No. 2, pp. 47-62.

[13] Leonardi, P. (2007). Activating the informational capabilities of information technology in organizations. Organization Science, Vol. 18, pp. 813-831

[14] Li, Q., C-Y Lu, S. (2008). Collaborative tagging applications and approaches. IEEE Multimedia, Vol. 15, pp. 14-21.

[15] Libert, B., Spector, J. (2007). We Are Smarter Than Me. How to Unleash the Power of Crowds in Your Business. $1^{\text {st }}$ ed. Wharton School publishing.

[16] Mader, S. (2007). Wikipatterns. Weily Publishing, Inc., Indianapolis, Indiana.

[17] McAfee, A. (2006). Enterprise 2.0: The Dawn of Emergent Collaboration. MITSloan Management review. Vol. 47, pp. 21-28.

[18] Nahapiet, J., Ghoshal, S. (1998). Social Capital, Intellectual Capital, and the Organizational Advantage. The Academy of Management Review, Vol. 23, No. 2 (Apr., 1998), pp. 242-266.

[19] Nardi, B. (1992). Studying context: A comparison of activity theory, situated action models and distributed cognition. Proceedings East-West Conference on Human-Computer Interaction. August 4-8, St. Petersburg, Russia. pp. 352-359.

[20] O'Reilly, T. (2005). Web 2.0: Compact definition - O'Reilly

Radar. [Online]. Available at: http://radar.oreilly.com/archives/2005/10/web-20

compactdefinition.html [Accessed 15 January 2009]

[21] Rogers, Y. (2006) Distributed Cognition and Communication. In The Encyclopedia of Language and Linguistics 2nd Edition. Oxford, pp. 181-202.

[22] Smith, E. (2008). Social relationships and groups: New insights on embodied and distributed cognition. Cognitive Systems Research, Vol. 9, pp. 24-32.

[23] Stahl, G. (2005). Group cognition in computer-assisted collaborative learning. Journal of Computer Assisted Learning, Vol. 21, No. 2. (April 2005), pp. 79-90.

[24] Surowiecki, J. (2005). The Wisdom of Crowds: Why the Many Are Smarter Than the Few. Abacus, London, UK.

[25] Tapscott, D., Williams, A. (2006). Wikinomics: How Mass Collaboration Changes Everything. Penguin Group, Inc., New York, N. Y.

[26] Touzet, J. (n.d.). Distributed cognition or intelligence? Patterns of group thought within online graduate-level coursework. Paper submitted for OISE/UT CTL1608F06

[27] Wang2, F., Zeng, D., Carley, K., Mao, W. (2007). Social computing: from social informatics to social intelligence. IEEE Computer Society, Vol. 22, pp. 79-83.

[28] Wasko, M., Faraj, S. (2005). Why should I Share? Examining Social Capital and Knowledge Contribution in Electronic Networks of Practice. MIS Quarterly, Vol. 29 No. 1, pp. 35-57/March 2005.

[29] Wood, L. (2005). Blogs \& Wikis: Technologies for enterprise applications?. The Glibane Report. Vol. 12, 2-9.

[30] Yukihiro, K. (2007). In-house use of Web 2.0: Enterprise 2.0. NEC Technical Journal. Vol. 2, p. 46-49. 